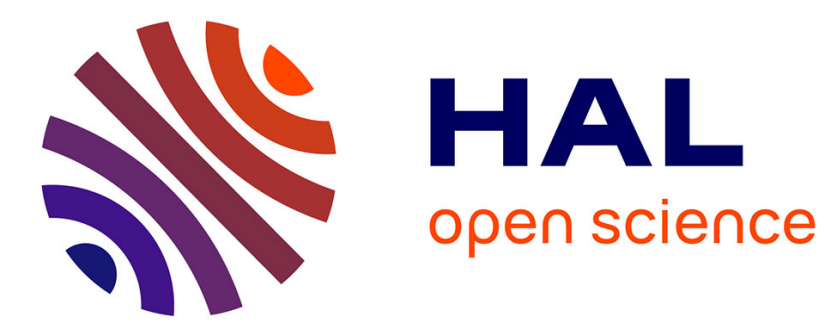

\title{
Line Directed Hypergraphs
}

Jean-Claude Bermond, Fahir Ergincan, Michel Syska

\section{To cite this version:}

Jean-Claude Bermond, Fahir Ergincan, Michel Syska. Line Directed Hypergraphs. Cryptoquater2011 ,Festchrift Jean-jacques," Springer verlag, pp.1-11, 2011, Lecture Notes in Computer Science, 6805. hal-00643785

\section{HAL Id: hal-00643785 https://hal.science/hal-00643785}

Submitted on 22 Nov 2011

HAL is a multi-disciplinary open access archive for the deposit and dissemination of scientific research documents, whether they are published or not. The documents may come from teaching and research institutions in France or abroad, or from public or private research centers.
L'archive ouverte pluridisciplinaire HAL, est destinée au dépôt et à la diffusion de documents scientifiques de niveau recherche, publiés ou non, émanant des établissements d'enseignement et de recherche français ou étrangers, des laboratoires publics ou privés. 


\title{
Line Directed Hypergraphs
}

\author{
Jean-Claude Bermond ${ }^{1}$, Fahir Ergincan ${ }^{2}$, and Michel Syska ${ }^{1}$ \\ 1 MASCOTTE, joint project CNRS-INRIA-UNS, \\ 2004 Route des Lucioles, BP 93, F-06902 Sophia-Antipolis, France \\ \{Jean-Claude.Bermond, Michel.Syska\}@inria.fr \\ 2 Ericsson Canada Inc. \\ 3500 Carling Av., Ottawa, Ontario, Canada \\ fahir.ergincan@ericsson.com
}

\begin{abstract}
In this article we generalize the concept of line digraphs to line dihypergraphs. We give some general properties in particular concerning connectivity parameters of dihypergraphs and their line dihypergraphs, like the fact that the arc connectivity of a line dihypergraph is greater than or equal to that of the original dihypergraph. Then we show that the De Bruijn and Kautz dihypergraphs (which are among the best known bus networks) are iterated line digraphs. Finally we give short proofs that they are highly connected.
\end{abstract}

Keywords: Hypergraphs, Line Hypergraphs, De Bruijn and Kautz networks, Connectivity

\section{Introduction}

In the beginning of the 80 's one of the authors - JCB - started working on the design of bus networks in order to answer a problem asked by engineers of the French telecommunications operator France Telecom. At that time he met JeanJacques (JJQ) who was working for Philips Research Labs and knew well how to design networks. Jean-Jacques kindly shared his knowledge and experience in particular on de Bruijn and Kautz networks and their generalizations. It was the birth of a fruitful and friendly collaboration on the topic of designing classical networks by using various tools of graph theory (see for example $[2-5,7]$ ). In the 90's, following ideas of JJQ, we extended the de Bruijn and Kautz digraphs to dihypergraphs, generalizing both their alphabetical and arithmetical definitions. There is another definition of de Bruijn and Kautz digraphs (see [11]) based on the fact that they are iterated line digraphs. This is useful to prove results using induction. We generalized this definition and used it in an unpublished manuscript (first version in 1993) which was announced in [6]). Unfortunately, this manuscript was never completely finished and never published. However, the results included have been used and some of them generalized in $[9,10]$.

Hypergraphs and dihypergraphs are used in the design of optical networks [15]. In particular, De Bruijn and Kautz hypergraphs have several properties that are beneficial in the design of large, dense, robust networks. They have 
been proposed as the underlying physical topologies for optical networks, as well as dense logical topologies for Logically Routed Networks (LRN) because of ease of routing, load balancing, and congestion reduction properties inherent in de Bruijn and Kautz networks. More recently, Jean-Jacques brought to our attention the web site (http://punetech.com/building-eka-the-worlds-fastestprivately-funded-supercomputer/) where it is explained how hypergraphs and the results of [6] were used for the design of the supercomputer EKA (http: //en.wikipedia.org/wiki/EKA_(supercomputer)).

Hence, when thinking to write an article in honor of JJQ, it was natural to exhume this old manuscript and to publish it hoping it will stimulate further studies and applications. Finally, that might convince Jean-Jacques that it is never too late to publish the valuable results he has obtained in his French thèse d'Etat in 1987 and that he had promised to JCB a long time ago.

The paper is organized as follows. We recall basic definitions of dihypergraphs in Section 2 and give the definition and first results on line dihypergraphs in Section 3. Then, in Section 4 we give connectivities properties of hypergraphs and in particular we prove that the arc connectivity of a line dihypergraph is greater than or equal to that of the original dihypergraph. We recall in Section 5 the arithmetical definition of de Bruijn and Kautz dihypergraphs and show that they are iterated line dihypergraphs. Finally, we use this property in Section 6 to determine their connectivities.

\section{Directed Hypergraphs}

A directed hypergraph (or dihypergraph) $H$ is a pair $(\mathcal{V}(H), \mathcal{E}(H))$ where $\mathcal{V}(H)$ is a non-empty set of elements (called vertices) and $\mathcal{E}(H)$ is a set of ordered pairs of non-empty subsets of $\mathcal{V}(H)$ (called hyperarcs). If $E=\left(E^{-}, E^{+}\right)$is a hyperarc in $\mathcal{E}(H)$, then the non-empty vertex sets $E^{-}$and $E^{+}$are called the in-set and the out-set of the hyperarc $E$, respectively. The sets $E^{-}$and $E^{+}$need not be disjoint. The hyperarc $E$ is said to join the vertices of $E^{-}$to the vertices of $E^{+}$. Furthermore, the vertices of $E^{-}$are said to be incident to the hyperarc $E$ and the vertices of $E^{+}$are said to be incident from $E$. The vertices of $E^{-}$ are adjacent to the vertices of $E^{+}$, and the vertices of $E^{+}$are adjacent from the vertices of $E^{-}$.

If $E$ is a hyperarc in a dihypergraph $H$, then $\left|E^{-}\right|$is the in-size and $\left|E^{+}\right|$ is the out-size of $E$ where the vertical bars denote the cardinalities of the sets. The maximum in-size and the maximum out-size of $H$ are respectively:

$$
s^{-}(H)=\max _{E \in \mathcal{E}(H)}\left|E^{-}\right| \text {and } s^{+}(H)=\max _{E \in \mathcal{E}(H)}\left|E^{+}\right| .
$$

The order of $H$ is the number of vertices in $\mathcal{V}(H)$ and is denoted by $n(H)$. The number of hyperarcs in $H$ is denoted by $m(H)$. We note that a digraph is a directed hypergraph $G=(\mathcal{V}(G), \mathcal{E}(G))$ with $s^{-}(G)=s^{+}(G)=1$.

Let $v$ be a vertex in $H$. The in-degree of $v$ is the number of hyperarcs that contain $v$ in their out-set, and is denoted by $d_{H}^{-}(v)$. Similarly, the out-degree of 
vertex $v$ is the number of hyperarcs that contain $v$ in their in-set, and is denoted by $d_{H}^{+}(v)$.

To a directed hypergraph $H$, we associate the bipartite digraph called the bipartite representation digraph of $H$ :

$$
R(H)=\left(\mathcal{V}_{1}(R) \cup \mathcal{V}_{2}(R), \mathcal{E}(R)\right) .
$$

A vertex of $\mathcal{V}_{1}(R)$ represents a vertex of $H$, and a vertex of $\mathcal{V}_{2}(R)$ a hyperarc of $H$. The arcs of $R(H)$ correspond to the incidence relation between the vertices and the hyperarcs of $H$. In other words, vertex $v_{i}$ is joined by an arc to vertex $e_{j}$ in $R(H)$, if $v_{i} \in E_{j}^{-}$in $H$; and vertex $e_{j}$ is joined by an arc to vertex $v_{k}$, if $v_{k} \in E_{j}^{+}$in $H$. This representation appears to be useful to draw a dihypergraph. For the ease of readability and to show the adjacency relations we duplicate the set $\mathcal{V}_{1}(R)$ and represent the $\operatorname{arcs}$ from $\mathcal{V}_{1}(R)$ to $\mathcal{V}_{2}(R)$ (adjacencies from vertices to hyperarcs) in the left part and the arcs from $\mathcal{V}_{2}(R)$ to $\mathcal{V}_{1}(R)$ in the right part.

Figure 1 shows an example of the de Bruijn dihypergraph $G B_{2}(2,6,3,4)$ (see Section 5 for the definition) with $|\mathcal{V}|=6,|\mathcal{E}|=4$. For each edge $E,\left|E^{-}\right|=$ $\left|E^{+}\right|=3$ and for each vertex $v,\left|d^{-}(v)\right|=\left|d^{+}(v)\right|=2$. Another example with 36 vertices and 24 edges is given in Figure 2 .

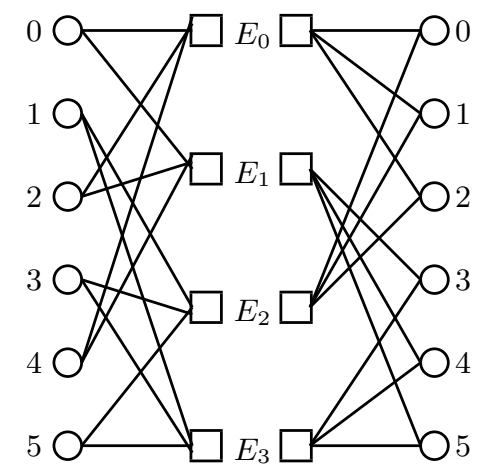

Fig. 1. Bipartite representation of $G B_{2}(2,6,3,4)$

If $H$ is a directed hypergraph, its dual $H^{*}$ is defined as follows: for every hyperarc $E \in \mathcal{E}(H)$ there is a corresponding vertex $e \in \mathcal{V}\left(H^{*}\right)$, and for every vertex $v \in \mathcal{V}(H)$ there is a corresponding hyperarc $V=\left(V^{-}, V^{+}\right) \in \mathcal{E}\left(H^{*}\right)$. Vertex $e$ is in $V^{-}$if and only if $v \in E^{+}$and similarly, $e$ is in $V^{+}$if and only if $v \in E^{-}$. Note that $R\left(H^{*}\right)$ is isomorphic to $R(H)$ (the roles of $\mathcal{V}_{1}(R)$ and $\mathcal{V}_{2}(R)$ being exchanged).

The underlying digraph of a directed hypergraph $H=(\mathcal{V}(H), \mathcal{E}(H))$ is the digraph $U(H)=(\mathcal{V}(U(H)), \mathcal{E}(U(H)))$ where $\mathcal{V}(U(H))=\mathcal{V}(H)$ and $\mathcal{E}(U(H))$ is the multiset of all ordered pairs $(u, v)$ such that $u \in E^{-}, v \in E^{+}$for some 
hyperarc $E \in \mathcal{E}(H)$. We emphasize that $U(H)$ needs not be simple: the number of arcs from $u$ to $v$ in $U(H)$ is the number of hyperarcs $E=\left(E^{-}, E^{+}\right)$in $H$ such that $u \in E^{-}$and $v \in E^{+}$. Thus, the in- and out-degrees of a vertex in $U(H)$ are

$$
d_{U(H)}^{-}(u)=\sum_{\substack{E \in \mathcal{E}(H) \\ E^{+} \ni u}}\left|E^{-}\right| \quad \text { and } \quad d_{U(H)}^{+}(u)=\sum_{\substack{E \in \mathcal{E}(H) \\ E^{-} \ni u}}\left|E^{+}\right| .
$$

\section{$3 \quad$ Line Directed Hypergraphs}

If $G$ is a digraph, we define its line digraph $L(G)$, as follows. An $\operatorname{arc} E=(u, v)$ of $G$ is represented by a vertex in $L(G)$, that we denote $(u E v)$; this notation is redundant but useful in order to generalize the concept to dihypergraphs. Vertex $(u E v)$ is adjacent to vertex $(w F y)$ in $L(G)$ if and only if $v=w$.

We now generalize the line digraph transformation to directed hypergraphs. Let $H=(\mathcal{V}, \mathcal{E})$ be a directed hypergraph, then the vertex set and the hyperarc set of its line directed hypergraph (denoted line dihypergraph), $L(H)$, are the following:

$$
\begin{aligned}
& \mathcal{V}(L(H))=\bigcup_{E \in \mathcal{E}(H)}\left\{(u E v) \mid u \in E^{-}, v \in E^{+}\right\}, \\
& \mathcal{E}(L(H))=\bigcup_{v \in \mathcal{V}(H)}\left\{(E v F) \mid v \in E^{+} \cap F^{-}\right\} ;
\end{aligned}
$$

where the in-set and the out-set of hyperarc $(E v F)$ are defined as :

$$
\begin{aligned}
& (E v F)^{-}=\left\{(u E v) \mid u \in E^{-}\right\}, \\
& (E v F)^{+}=\left\{(v F w) \mid w \in F^{+}\right\} .
\end{aligned}
$$

Figure 2 shows the line dihypergraph $L\left[G B_{2}(2,6,3,4)\right]$ of the hypergraph of Figure 1. Note that if $G$ is a digraph, then $L(G)$ is exactly the line digraph of $H$. The following theorems give some relations implying the functions previously defined. The proofs are straightforward and omitted.

Theorem 1. The digraphs $R(L(H))$ and $L^{2}(R(H))$ are isomorphic.

Proof. The vertices of $L^{2}(R(H))$ correspond to the paths of length 2 in $R(H)$ and are of the form $u E v$ (representing the vertices of $L(H)$ ) or $E v F$ (representing the edges of $L(H)$ ).

Theorem 2. The digraphs $U(L(H))$ and $L(U(H))$ are isomorphic.

Theorem 3. The digraphs $(L(H))^{*}$ and $L\left(H^{*}\right)$ are isomorphic.

In a first version of this article, we conjectured the following characterization of the line directed hypergraphs. This conjecture has been proved in [10]. 
Theorem 4. [10] $H$ is a line directed hypergraph if and only if the underlying multidigraph $U(H)$ is a line digraph, and the underlying multidigraph of the dual $U\left(H^{*}\right)$ is a line digraph.

\section{Connectivity}

A dipath in $H$ from vertex $u$ to vertex $v$ is an alternating sequence of vertices and hyperarcs $u=v_{0}, E_{1}, v_{1}, E_{2}, v_{2}, \cdots, E_{k}, v_{k}=v$ such that vertex $v_{i-1} \in E_{i}^{-}$ and $v_{i} \in E_{i}^{+}$for each $1 \leq i \leq k$.

A dihypergraph $H$ is strongly connected if there exists at least one dipath from each vertex to every other vertex. Otherwise it is disconnected. The vertexconnectivity, $\kappa(H)$, of a dihypergraph is the minimum number of vertices to be removed to obtain a disconnected or trivial dihypergraph (a dihypergraph with only one vertex). Similarly, the hyperarc connectivity, $\lambda(H)$, of a (non-trivial) dihypergraph is the minimum number of hyperarcs to be removed to obtain a disconnected dihypergraph.

Any two dipaths in $H$ are vertex disjoint if they have no vertices in common except possibly their end vertices, and are hyperarc disjoint if they have no hyperarc in common. The theorem of Menger [13] establishes that the vertex (resp. arc) connectivity of a graph is $\kappa$ if and only if there exist at least $\kappa$ vertex (resp. arc) disjoint paths between any pair of vertices. This relation also holds true for dihypergraphs. It is an easy matter to show this by adapting Ore's proof ([14], pp. 197-205) of Menger's theorem to dihypergraphs.

Let denote by $\delta(H)$ the minimum degree of $H$ and by $s(H)$ the minimum of the in-size and out-size of $H$. That is $\delta(H)=\min _{v \in \mathcal{V}(H)}\left(d_{H}^{-}(v), d_{H}^{+}(v)\right)$ and $s(H)=\min \left(s^{-}(H), s^{+}(H)\right)$. The two results of Proposition 1 are immediate.

\section{Proposition 1.}

$$
\begin{gathered}
\kappa(H)=\kappa(U(H)) \\
\lambda(H) \leq \delta(H)
\end{gathered}
$$

The generalization of the relation $\kappa(G) \leq \lambda(G)$ for a digraph (case $s(H)=1$ ) is as follows.

Theorem 5. If $n \geq(\lambda(H)+1) s(H)+1$, then $\kappa(H) \leq \lambda(H) s(H)$.

Proof. In this proof let $\lambda=\lambda(H)$ and $s=s(H)$. Let $\Lambda$ be a cut set of $\lambda$ hyperarcs disconnecting $H$. Let $A$ and $B$ be two non empty sets of vertices such that $A \cup B=\mathcal{V}(H)$ and there is no dipath from $A$ to $B$ in $H-\Lambda$.

Let $|A|=p s+\alpha, 1 \leq \alpha \leq s,|B|=q s+\beta, 1 \leq \beta \leq s$.

As $|A|+|B|=n=(p+q) s+\alpha+\beta$, if $p+q \leq \lambda-1$ then we get $n \leq$ $(\lambda-1) s+2 s=(\lambda+1) s:$ a contradiction. So $p+q \geq \lambda$.

Choose $p^{\prime} \leq p$ and $q^{\prime} \leq q$ such that $p^{\prime}+q^{\prime}=\lambda$. Let $A^{\prime}$ be the set of in vertices of $p^{\prime}$ hyperarcs of $\Lambda$ and $B^{\prime}$ the set of out vertices of the $q^{\prime}=\lambda-p^{\prime}$ other hyperarcs of $\Lambda .\left|A^{\prime}\right| \leq p^{\prime} s$ and $\left|B^{\prime}\right| \leq q^{\prime} s$. 


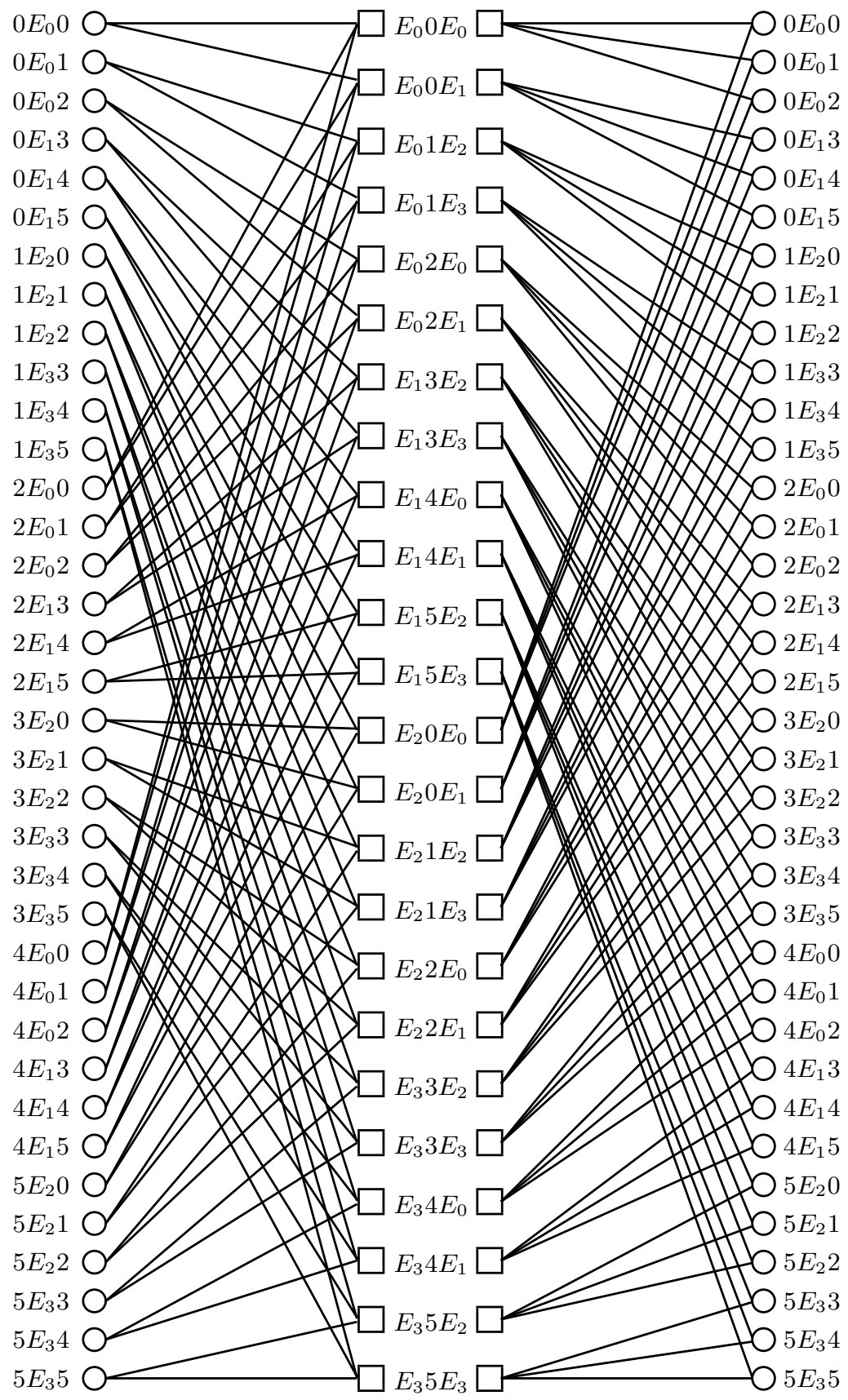

Fig. 2. Bipartite representation of $L\left[G B_{2}(2,6,3,4)\right]=G B_{2}(2,36,3,24)$ 
So, as $|A|>p s \geq p^{\prime} s$ there exists a vertex $u$ in $A-A^{\prime}$ and similarly, as $|B|>q s \geq q^{\prime} s$ there exists a vertex $v$ in $B-B^{\prime}$. There is no dipath from $u$ to $v$ in $\mathcal{V}(H)-A^{\prime}-B^{\prime}$. So, $A^{\prime} \cup B^{\prime}$ is a disconnecting set of cardinality less or equal $\left(p^{\prime}+q^{\prime}\right) s=\lambda s$. Therefore $\kappa(H) \leq \lambda s$.

This bound is the best possible. Consider the hypergraph on $n=(\lambda+1) s$ vertices with vertex set $A \cup B$ with $A=\left\{a_{1}, \ldots, a_{\lambda s}\right\}$ and $B=\left\{b_{1}, \ldots, b_{s}\right\}$. Let the hyperarcs from $A$ to $B$ be $E_{i}=\left(\left\{a_{(i-1) s+1}, \ldots, a_{i s}\right\},\left\{b_{1}, \ldots, b_{s}\right\}\right), 1 \leq i \leq \lambda$. Let furthermore put an hyperarc for every possible ordered pair $\left(a_{i}, a_{j}\right),\left(b_{i}, b_{j}\right)$, and $\left(b_{i}, a_{j}\right)$. Clearly, if we delete the $\lambda$ hyperarcs $E_{i}$, we disconnect $A$ from $B$ and so $\lambda(H) \leq \lambda$. However, deleting any set of vertices leaves a strongly connected hypergraph, so $\kappa(H)=(\lambda+1) s-1$.

Corollary 1. Let $H$ be a dihypergraph with $n \geq \delta s+1$. If $\kappa(H) \geq(d-1) s+1$, then $\lambda(H)=\delta$.

Proof. We know that $\lambda(H) \leq \delta$. Suppose $\lambda(H) \leq \delta-1$, then by theorem 5 $\kappa(H) \leq(\delta-1) s$, a contradiction.

Theorem 6. $\lambda(H) \geq \min \left(\delta(H), \kappa\left(H^{*}\right)\right)$.

Proof. Suppose that $\lambda(H)<\delta(H)$ and $\lambda(H)<\kappa\left(H^{*}\right)$. Let $\Lambda$ be a set of hyperarcs with $|\Lambda|=\lambda(H)$. Let $H^{\prime}=H-\Lambda$. We will show that $H^{\prime}$ remains connected. Let $u$ and $v$ be any couple of vertices. As $\lambda(H)<\delta(H)$, vertex $u$ is in the in-set of some hyperarc $E$ of $H^{\prime}$. Similarly, $v$ is in the out-set of some hyperarc $F$ of $H^{\prime}$. As $\lambda(H)<\kappa\left(H^{*}\right)$, there exists in $H^{\prime *}$ a dipath from $E$ to $F$. So, there exists in $H^{\prime}$ a dipath from $u$ to $v$.

Theorem 7. If $|\mathcal{V}(H)|>\lambda(H), \lambda(L(H)) \geq \lambda(H)$.

Proof. Let $\lambda(L(H))=\lambda$ and let $C$ be a cut with $\lambda$ hyperarcs of $L(H)$ consisting of the $\lambda$ hyperarcs $E_{i} v_{i} F_{i}, 1 \leq i \leq \lambda$ and separating the set of vertices $S$ and $\bar{S}$ with $S \neq \emptyset, \bar{S} \neq \emptyset$ and $S \cup \bar{S}=\mathcal{V}(L(H))$. We will show that in $H$ there exists a cut with $\lambda$ hyperarcs, so $\lambda(H) \leq \lambda=\lambda(L(H))$.

Consider in $H$ the set of hyperarcs $E_{i}, 1 \leq i \leq \lambda$. Either it is a cut and we are done; otherwise $H-\left\{E_{i}, 1 \leq i \leq \lambda\right\}$ is connected. Let $u E v$ be a vertex of $S$ in $L(H)$ and $x F y$ a vertex of $\bar{S}$ in $L(H)$. Note that $x$ may be equal to $v$. As $H-\left\{E_{i}, 1 \leq i \leq \lambda\right\}$ is connected, there exists a dipath from $v$ to $x$ in $H$ - a circuit if $x=v-P=v, E_{\lambda+1}, v_{\lambda+1}, \ldots, E_{\lambda+j}, v_{\lambda+j}, \ldots, E_{p}, x$ with the $E_{\lambda+j} \neq E_{i}$. This dipath induces in $L(H)$ the dipath $Q$ from $u E v$ to $x F y$. $Q=u E v, E v E_{\lambda+1}, v E_{\lambda+1} v_{\lambda+1}, \ldots, E_{\lambda+j} v_{\lambda+j} E_{\lambda+j+1}, \ldots, E_{p} x F, x F y . C$ being a cut, then one of the hyperarcs of $Q$ should belong to $C$; but the hyperarcs of the path are all different from the hyperarcs $E_{i} v_{i} F_{i}, 1 \leq i \leq \lambda$ of the cut except possibly for the first arc when $E=E_{i}$ and $v=v_{i}$ (and $E_{\lambda+1}=F_{i}$ ) for some $i$. So, we deduce that all the vertices of $\mathrm{S}$ are of the form $u E_{i} v_{i}$.

Similarly, if we consider in $H$ the set $F_{i}, 1 \leq i \leq \lambda$ we get either a cut in $H$ of size $\lambda$ or the fact that all vertices of $\bar{S}$ are of the form $v_{i} F_{i} y$.

As $S \cup \bar{S}=\mathcal{V}(L(H))$, we get that any vertex different from $v_{i}$ has in or out-degree less or equal $\lambda$. So, either $\lambda(H) \leq \lambda$ or $H$ contains only the vertices $v_{i}, 1 \leq i \leq \lambda$, and so $|\mathcal{V}(H)| \leq \lambda$, contradicting the hypothesis. 


\section{Generalized De Bruijn and Kautz Dihypergraphs}

In [1] different generalizations of de Bruijn or Kautz digraphs have been proposed. We will show that they are line dihypergraphs. Due to lack of space, we deal here only with the "arithmetical definition" and do not give all the details.

Let $d, n, m, s$ be 4 integers satisfying:

$$
\begin{aligned}
d n & \equiv 0 & & (\bmod m) \\
s m & \equiv 0 & & (\bmod n) .
\end{aligned}
$$

The generalized de Bruijn dihypergraph $G B_{2}(d, n, s, m)$ with $n$ vertices, $m$ hyperarcs, out-degree $d$ and out-size $s$ is defined as follows.

The vertices are the integer modulo $n$ and the hyperarcs are the integer modulo $m$. Vertex $i$ is incident to the hyperarcs $E_{j}$ with $j \equiv d i+\alpha(\bmod m)$, $0 \leq \alpha \leq d-1$. That is : $\Gamma^{+}(i)=\left\{E_{j}, j \equiv d i+\alpha(\bmod m), 0 \leq \alpha \leq d-1\right\}$

The out-set of the hyperarc $E_{j}$ contains the vertices $i \equiv s j+\beta(\bmod n)$, $0 \leq \beta \leq s-1$. That is : $E_{j}^{+}=\{i, i \equiv s j+\beta(\bmod n), 0 \leq \beta \leq s-1\}$

Figure 1 shows $G B_{2}(2,6,3,4)$ and Figure 2 shows $G B_{2}(2,36,3,24)$.

Note that condition (1) is needed in order to insure that $i$ and $i+n$ have the same out-neighborhood. Indeed, $d(i+n)+\alpha=d i+\alpha+d n \equiv d i+\alpha(\bmod n)$ if and only if $d n \equiv 0(\bmod m)$. Similarly condition $(2)$ is needed to insure that $E_{j}^{+}=E_{j+m}^{+}$as $s(j+m)+\beta=s j+\beta+s m \equiv s j+\beta(\bmod m)$ if and only if $s m \equiv 0(\bmod n)$.

In [1] it is shown that $\left|E^{-}\right|=\frac{d n}{m}, d^{-}(v)=\frac{s m}{n}$. In particular, if $d n=s m$, then $G B_{2}(d, n, s, m)$ is regular (with $d^{-}=d^{+}=d$ ) and uniform $\left(s^{-}=s^{+}=s\right.$ ).

It is also proved that $U\left[G B_{2}(d, n, s, m)\right]=G B(d s, n)$, the generalized de Bruijn digraph whose vertices are the integer modulo $n$, vertex $i$ being connected to vertices $d s i+\alpha, 0 \leq \alpha \leq d s-1$.

One motivation to introduce de Bruijn dihypergraphs was their good diameter which is of the order of the Moore bound for directed hypergraphs. In [1] it is shown that the diameter of $G B_{2}(d, n, s, m)$ is $\left\lceil\log _{d s} n\right\rceil$. In particular, when $n=(d s)^{D}$ and $m=d^{2}(d s)^{D-1}$, the diameter is exactly $D$ and these dihypergraphs are the standard de Bruijn dihypergraphs.

Theorem 8. $L\left[G B_{2}(d, n, s, m)\right]$ is isomorphic to $G B_{2}(d, d s n, s, d s m)$.

Proof. Let $H=L\left[G B_{2}(d, n, s, m)\right]$ and $H^{\prime}=G B_{2}(d, d s n, s, d s m)$.

A vertex of $H$ is of the form $i E_{j} i^{\prime}$ with $j \equiv d i+a(\bmod m)$, for some $a$, $0 \leq a \leq d-1$ and $i^{\prime} \equiv s j+b(\bmod n)$, for some $b, 0 \leq b \leq s-1$. A hyperarc of $H$ is of the form $E_{j} i^{\prime} E_{j^{\prime}}$ with $i^{\prime} \equiv s j+b(\bmod n), 0 \leq b \leq s-1$ and $j^{\prime} \equiv d i^{\prime}+a^{\prime}$ $(\bmod m)$, for some $a^{\prime}, 0 \leq a^{\prime} \leq d-1$.

Consider the mapping of $\mathcal{V}(H)$ on $\mathcal{V}\left(H^{\prime}\right)$ which associates to the vertex $i E_{j} i^{\prime}$ of $H$ the vertex of $H^{\prime}: d s i+s a+b$ and the mapping of $\mathcal{E}(\mathcal{H})$ on $\mathcal{E}\left(\mathcal{H}^{\prime}\right)$ wich associates to $E_{j} i^{\prime} E_{j^{\prime}}$ the hyperarc of $H^{\prime} d s j+d b+a^{\prime}$.

Clearly, these mappings are one-to-one. Furthermore, they preserve the adjacency relations. Indeed, vertex $i E_{j} i^{\prime}$ is incident in $H$ to the hyperarcs $E_{j} i^{\prime} E_{j^{\prime}}$ where $j^{\prime}=d i^{\prime}+\alpha$, for $\alpha=0,1, \ldots, d-1$. Its associated vertex in $H^{\prime}, d s i+s a+b$ 
is incident in $H^{\prime}$ to the hyperarcs $E_{k}$ where $k=d(d s i+s a+b)+\alpha$, for $\alpha=0,1, \ldots, d-1$. But the images of the hyperarcs $E_{j} i^{\prime} E_{j^{\prime}}$ are the hyperarcs of $H^{\prime}$ of the form $d s j+d b+\alpha=d s(d i+a)+d b+\alpha=d[d s i+s a+b]+\alpha$. They are exactly the neighbors of $d s i+s a+b$.

Similarly, the out set of $E_{j} i^{\prime} E_{j^{\prime}}$ consists of the vertices $i^{\prime} E_{j^{\prime}} i^{\prime \prime}$ with $i^{\prime \prime}=$ $s j^{\prime}+\beta$, for $\beta=0,1, \ldots, s-1$. The image of $E_{j} i^{\prime} E_{j^{\prime}}$ in $H^{\prime}$ is the hyperarc $d s j+d b+a^{\prime}$ which has as out set the vertices $s\left[d s j+d b+a^{\prime}\right]+\beta$ for $\beta=$ $0,1, \ldots, s-1$. But the images of the vertices of $H, i^{\prime} E_{j^{\prime}} i^{\prime \prime}$ are the vertices $d s i^{\prime}+s a^{\prime}+\beta=d s[s j+b]+s a^{\prime}+\beta=s\left[d s j+d b+a^{\prime}\right]+\beta$, that is exactly the outset of the hyperarc $d s j+d b+a^{\prime}$.

Generalized Kautz dihypergraphs $G K(d, n, s, m)$ are defined similarly except for the outset of an hyperarc $E_{j}$ which contains the vertices $-s j-\beta$ for $1 \leq \beta \leq s$.

One can prove similarly as for $G B_{2}(d, n, s, m)$ that:

Theorem 9. $L[G K(d, n, s, m)]$ is isomorphic to $G K(d, d s n, s, d s m)$.

\section{Connectivity of De Bruijn and Kautz dihypergraphs}

The vertex connectivity of the generalized de Bruijn and Kautz dihypergraphs follows easily from Proposition 1. Indeed their underlying digraphs are generalized de Bruijn (resp Kautz) digraphs whose connectivity is known. Therefore using the results of $[8,12]$ we get:

\section{Proposition 2.}

$$
\begin{aligned}
& \kappa\left[G B_{2}(d, n, s, m)\right]=d s-1 \\
& \kappa[G K(d, n, s, m)]= \begin{cases}d s, & \text { if } n \text { is a multiple of } d s+1 \text { and } \operatorname{gcd}(d s, n) \neq 1 ; \\
d s-1, & \text { otherwise. }\end{cases}
\end{aligned}
$$

As we will see the hyperarc connectivity of the generalized de Bruijn and Kautz dihypergraphs is equal to their minimum degree as soon as $s \geq 2$. The result was more difficult to prove. In the first version of this article we proved it for the de Bruijn and Kautz dihypergraphs of diameter $D$ (case $n=(d s)^{D}$ and $\left.m=d^{2}(d s)^{D-1}\right)$ by induction. Indeed it is easy to verify that the result is true for $D=1$; then we used the fact that these hypergraphs are iterated line dihypergraphs combined with Theorem 7 . We also had a very complicated proof for the generalized ones, but thanks to Theorem 6 we got a simpler proof.

Theorem 10. Let $H=G B_{2}(d, n, s, m)$ or $G K(d, n, s, m)$, with $s \geq 2$, then $\lambda(H)=\delta(H)$.

Proof. Let $H=G B_{2}(d, n, s, m)$ (respectively $G K(d, n, s, m)$ ), with $s \geq 2$. Then its dual is $H^{*}=G B_{2}(s, m, d, n)$ (respectively $G K(s, m, d, n)$ ) and so by proposition $2, \kappa\left(H^{*}\right) \geq d s-1>d \geq \delta(H)($ as $s \geq 2)$ and so by Theorem $6 \lambda(H) \geq \delta(H)$ and as by Proposition $1 \lambda(H) \leq \delta(H)$, we get the equality. 


\section{Conclusion}

In this article we have shown various properties of the line dihypergraphs like connectivities ones. We have also shown that de Bruijn and Kautz hypergraphs are iterated line dihypergraphs and have very good connectivity, reinforcing their attractiveness to build bus networks. Other vulnerability problems have been considered in [9] and generalization to partial line dihypergraphs has also been investigated in [10]. However, other properties might be worth of future investigations.

\section{References}

1. J.-C. Bermond, R.W. Dawes, and F.Ö. Ergincan. De Bruijn and Kautz bus networks. Networks, 30:205-218, 1997.

2. J.-C. Bermond, C. Delorme, and J.-J. Quisquater. Tables of large graphs with given degree and diameter. Inform. Process. Lett., 15(1):10-13, 1982.

3. J.-C. Bermond, C. Delorme, and J.-J. Quisquater. Grands graphes de degré et diamètre fixés. In Combinatorial mathematics (Marseille-Luminy, 1981), volume 75 of North-Holland Math.Stud., pages 65-73, Amsterdam, 1983. NorthHolland.

4. J.-C. Bermond, C. Delorme, and J.-J. Quisquater. Strategies for interconnection networks: some methods from graph theory. J. Parallel Distributed Computing, 3(4):433-449, 1986.

5. J.-C. Bermond, C. Delorme, and J.-J. Quisquater. Table of large $(\Delta, d)$-graphs. Discrete Applied Mathematics, 37/38:575-577, 1992.

6. J.-C. Bermond and F.Ö. Ergincan. Bus interconnection networks. Discrete Applied Mathematics, 68:1-15, 1996.

7. J.-C. Bermond, P. Hell, and J.-J. Quisquater. Construction of large packet radio networks. Parallel Processing Letters, 2(1):3-12, 1992.

8. J. Fàbrega and M. A. Fiol. Maximally connected digraphs. Journal of Graph Theory, 13(6):657-668, 1989.

9. D. Ferrero and C. Padró. Connectivity and fault-tolerance of hyperdigraphs. Discrete Applied Mathematics, 117:15-26, 2002.

10. D. Ferrero and C. Padró. Partial line directed hypergraphs. Networks, 39:61-67, 2002.

11. M.A. Fiol, J.L.A. Yebra, and I.A. De Miquel. Line digraph iterations and the (d, k) digraph problem. Computers, IEEE Transactions on, C-33(5):400-403, may 1984.

12. N. Homobono and C. Peyrat. Connectivity of Imase and Itoh digraphs. IEEE Transactions on Computers, 37:1459-1461, 1988.

13. K. Menger. Zur allgemeinen Kurventheorie. Fundamenta Mathematicae, 10:96$115,1927$.

14. O. Ore. Theory of Graphs, volume 38 of Colloquium Publications. American Mathematical Society, 1962.

15. T. E. Stern and K. Bala. Multiwavelength Optical Networks: A Layered Approach. Addison-Wesley Longman Publishing Co., Inc., Boston, MA, USA, 1999. 